\title{
Strained layer epitaxy of InGaAs by MBE and migration enhanced epitaxy - comparison of growth modes and surface quality
}

\author{
Y.C. Chen, P.K. Bhattacharya and J. Singh \\ Solid State Electronics Laboratory, Department of Electrical Engineering and Computer Science, The University of Michigan, Ann Arbor, \\ Michigan 48109-2122, USA
}

\begin{abstract}
Our measurements on a series of pseudomorphic n-type modulation doped field effect transistors have shown that the mobility of the two-dimensional carriers appears to suffer from increased interface roughness as the strain increases. Reflection high energy electron diffraction oscillation studies in $\mathrm{In}_{x} \mathrm{Ga}_{1-x}$ As grown by molecular beam epitaxy show that as the strain in the overlayer increases, the growth modes change from layer-by-layer to three-dimensional island growth. However, in migration enhanced epitaxy, we find that the growth remains in the layer-by-layer mode even for high strain. Reflection high energy electron diffraction oscillations also show that surface roughness in strained layers grown by molecular beam epitaxy can be smoothed by just a few monolayers grown by migration enhanced epitaxy. This suggests that for pseudomorphic devices most of the strained active layer might be grown by molecular beam epitaxy but just a few monolayers before interface formation by migration enhanced epitaxy to produce an abrupt interface. Our device results validate this observation.
\end{abstract}

\section{Introduction}

Increased efforts have concentrated on the growth of pseudomorphic heterostructure systems, including $\operatorname{In}_{x} \mathrm{Ga}_{1-x} \mathrm{As} / \mathrm{AlGaAs}$ (on GaAs substrates) and $\mathrm{In}_{0.53+x} \mathrm{Ga}_{0.47} \mathrm{As} / \mathrm{In}_{0.52} \mathrm{Al}_{0.48}$ As (on InP substrates). Modulation doped field effect transistors (MODFETs) based on these pseudomorphic heterostructures have several advantages over their lattice-matched counterparts [1,2]. Pseudomorphic MODFETs contain a highly strained pseudomorphic quantum well channel. It is extremely important to understand the epitaxial growth modes of such highly strained layers, since the growth modes control the growth front and interface roughness. The latter, in turn, controls the transport properties of the pseudomorphic channel and ultimately the device performance $[3,4]$. In this study we have investigated the suitability of molecular beam epitaxy (MBE), and its variation, commonly known as migration enhanced epitaxy (MEE), for the realization of high quality pseudomorphic heterostructures.

\section{Growth modes in molecular beam epitaxy}

In the growth of $\operatorname{In}_{x} \mathrm{Ga}_{1-x}$ As by $\mathrm{MBE}$, since atoms and molecules ( $\mathrm{Ga}$, In, $\mathrm{As}_{4}$ ) impinge randomly on the substrate, kinetics and thermodynamics both play important roles in establishing the growth modes. Thermodynamic parameters such as surface bond-strengths and substrate temperature (free energy $=$ internal energy $-T_{\text {sub }} \times$ entropy) decide whether in equilibrium the surface is atomically abrupt or three-dimensional in nature. Kinetic parameters (surface migration and evaporation) decide whether the thermodynamical equilibrium is reached. If the surface migration rate of the impinging atoms is high and thermodynamics favor an atomically abrupt surface, a layer-by-layer growth mode results. On the other hand if the surface kinetics are very small or the thermodynamics equilibrium state is not atomically flat, a three-dimensional growth mode will result [5]. These growth modes can be studied in-situ by reflection high energy electron diffraction (RHEED) oscillation studies [6,7]. In (100)- 

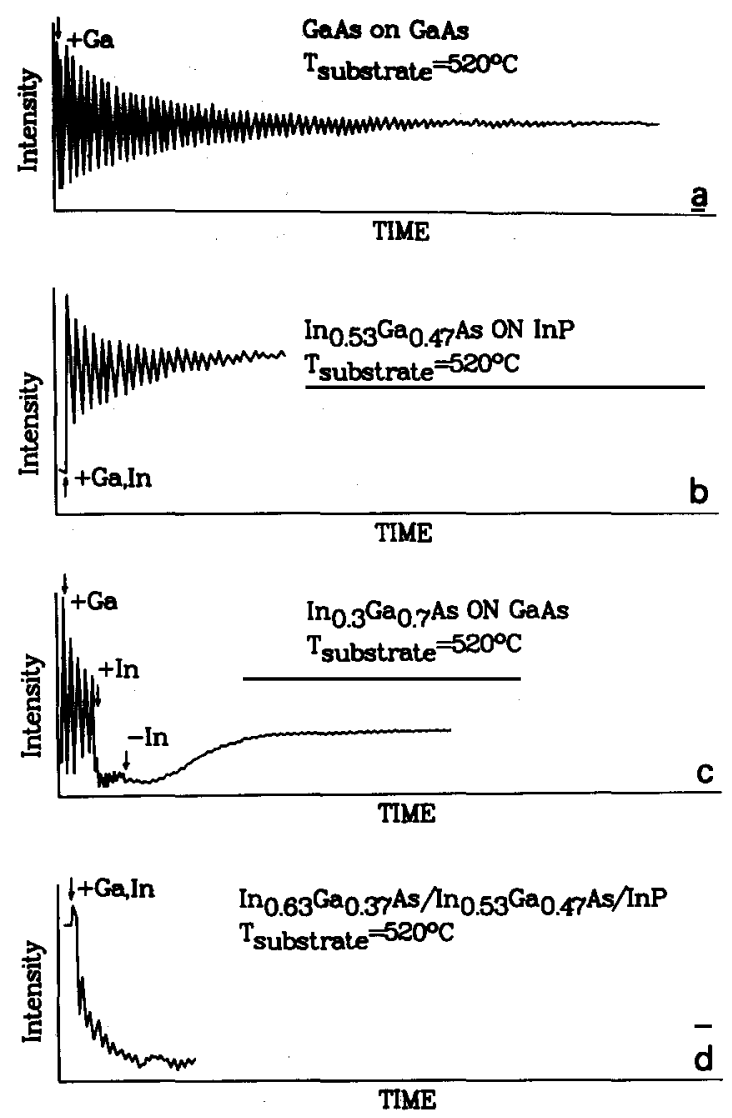

Fig. 1. RHEED oscillations observed during MBE growth of (a) GaAs on GaAs, (b) $\mathrm{In}_{0.53} \mathrm{Ga}_{0.47}$ As on InP, (c) $\mathrm{In}_{0.3} \mathrm{Ga}_{0.7}$ As on GaAs and (d) $\operatorname{In}_{0.63} \mathrm{Ga}_{0.37}$ As on InP.

oriented growth, the energy difference between an atomically abrupt and a rough surface results from second neighbor bondstrengths under the appropriate surface reconstruction. In absence of biaxial strain, if the second neighbor bond energy $\omega_{2}$ is larger than $k T_{\text {sub }}$, the equilibrium state is atomically abrupt and a layer-by-layer growth mode can result if the surface kinetics are sufficiently high [8]. In figs. $1 \mathrm{a}$ and $1 \mathrm{~b}$ we show RHEED oscillations for the lattice matched growth of GaAs and $\mathrm{In}_{0.53} \mathrm{Ga}_{0.47}$ As on InP. As can be seen from the sustained oscillations, layer-by-layer growth occurs.

In strained system, besides the second neighbor bond energy, $\omega_{2}$, one has to consider the strain energy in determining the thermodynamic equilibrium state. Using a simple model and energy minimization techniques we have shown that the surface prefers to be formed from islands rather than have an atomically flat profile and that the height (in monolayers) of the islands is given by

$n^{3} \cong 2 \frac{\omega_{1}}{\omega_{2}} \frac{R_{0}}{d_{c}}$

where $\omega_{1} / \omega_{2}$ is the ratio of the nearest to second neighbor bond energies, $R_{0}$ is the substrate lattice constant and $d_{\mathrm{c}}$ is the critical thickness. At critical thickness the strain energy equals the dislocation formation energy. For lattice matched system $\left(d_{\mathrm{c}} \rightarrow \infty\right), n$ goes to zero, i.e., to an atomically flat surface. However, as the value of $d_{\mathrm{c}}$ decreases (i.e., the lattice mismatch increases) we expect a three-dimensional surface. In figs. $1 \mathrm{c}$ and $1 \mathrm{~d}$ we have shown RHEED oscillation data for growth of strained $\mathrm{In}_{0.3} \mathrm{Ga}_{0.7}$ As on GaAs and InGaAs on InP, respectively. As can be seen from this figure, in presence of strain the layer grows in a $3 \mathrm{D}$ mode which is reflected by the abrupt decay of the RHEED oscillations.

\section{Migration enhanced epitaxy}

Since the minimum free energy for a strained system favors a 3D surface, the growth of strained systems cannot be improved by simply enhancing the surface migration rate of the adatoms. One expects the free energy to depend upon the surface reconstruction during growth. In MBE the surface

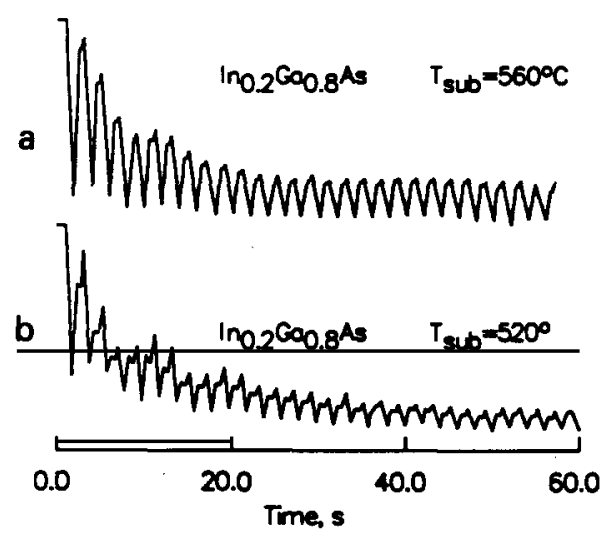

Fig. 2. RHEED oscillations observed during growth of $\mathrm{In}_{0.2}$ $\mathrm{Ga}_{0.8}$ As by MEE. 


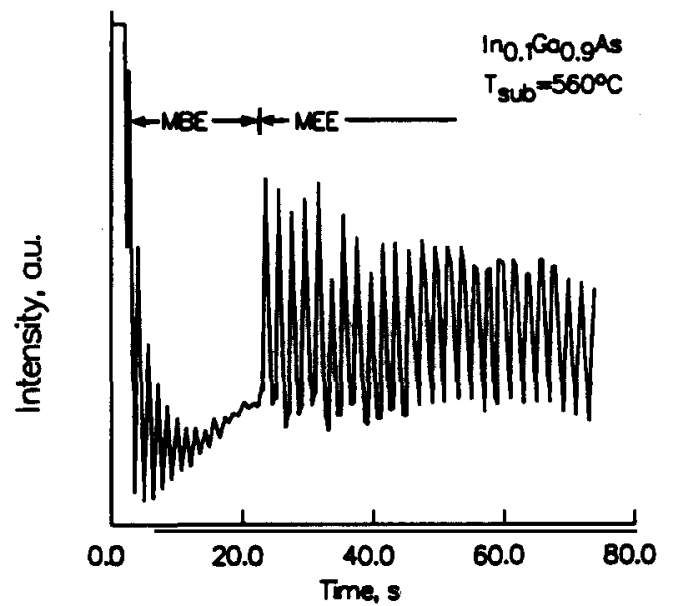

Fig. 3. RHEED oscillations observed during growth of $\operatorname{In}_{0.1}$ $\mathrm{Ga}_{0.9}$ As with first fifteen monolayers grown by MBE then followed immediately by MEE.

is anion stabilized with a $(2 \times 4)$ or $c(2 \times 8)$ reconstruction. It is therefore important to examine other possible surface reconstructions which might change the surface chemical energy. Since MBE of III-V semiconductors cannot be carried out under cation-rich conditions because the excess cation causes non-stoichiometric growth, a practical approach is migration enhanced epitaxy (MEE) $[9,10]$.

During MEE growth, group III and group V fluxes are delivered alternately by shutter control. The surface reconstruction thus alternates between cation and anion stabilized. If the shutter opening time is adjusted such that one monolayer of atoms are deposited in each shutter opening, layer-by-

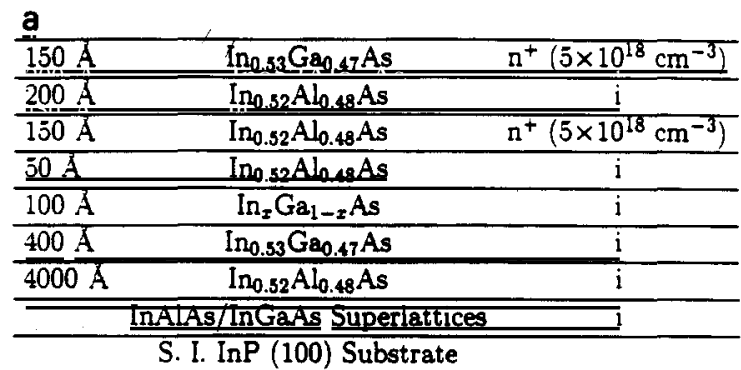

layer growth is possible, with long-lasting oscillations. Shown in fig. 2 are the observed RHEED oscillations for growth of $\mathrm{In}_{0.2} \mathrm{Ga}_{0.8}$ As by MEE. Differences in RHEED patterns between MBE and MEE are also observed for $\operatorname{In}_{x} \mathrm{Ga}_{1-x}$ As with $x=0.3$. In MBE growth, the RHEED pattern becomes spotty after a few monolayers are deposited. During MEE, the pattern remains streaked even after 20 monolayers, suggesting the growth is two-dimensional. RHEED studies also show that surface roughness in strained layers grown by MBE can be smoothed by just a few monolayers overgrown by MEE, as evidenced by the restoration of strong oscillations (fig. 3). This suggests that for pseudomorphic devices most of the strained active layer might be grown by MBE and just a few monolayers before interface formation by MEE to produce an abrupt interface.

\section{Transport properties and device performance}

We have carried out experiments on both GaAs-based and InP-based pseudomorphic MODFET heterostructures. We will first discuss the results obtained from InP-based structures grown by MBE. The schematic of a typical InPbased structure is shown in fig. 4a. The sheet electron density in all the samples varied in the range $(2.0 \times 3.2) \times 10^{12} \mathrm{~cm}^{-2}$. In fig. $4 \mathrm{~b}$ we show a plot of the 300 and $77 \mathrm{~K}$ mobilities versus excess In content in the channel. MODFETs, with 0.8 $\mu \mathrm{m}$ gate stripes, were fabricated on the hetero-

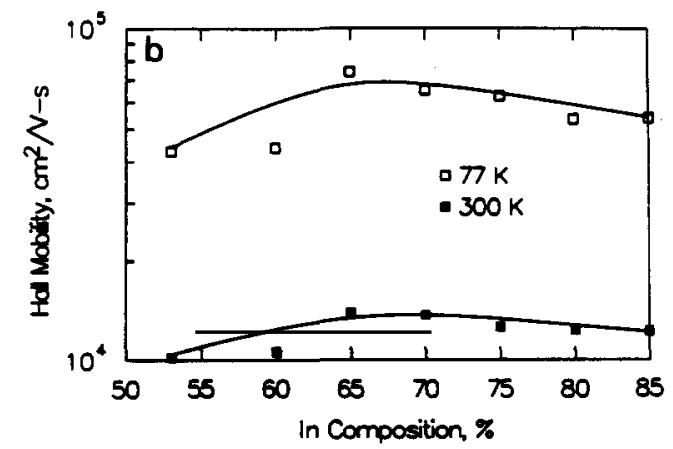

Fig. 4. (a) Schematic of MBE-grown n-type pseudomorphic MODFET on InP. (b) Hall mobilities of pseudomorphic MODFETs with increasing indium content in the channel at 77 and $300 \mathrm{~K}$. 
Table 1

DC and microwave characteristics of $0.8 \times 150 \mu \mathrm{m}$ gate MODFETs with pseudomorphic $\operatorname{In}_{x} \mathrm{Ga}_{1-x} \mathrm{As} / \mathrm{In}_{0.52} \mathrm{Al}_{0.48}$ As quantum wells

\begin{tabular}{lllll}
\hline$x$ & $\begin{array}{l}g_{\mathrm{m}, \max } \\
(\mathrm{mS} / \mathrm{mm})\end{array}$ & $\begin{array}{l}I_{\mathrm{ds}, \max } \\
(\mathrm{mA} / \mathrm{mm})\end{array}$ & $\begin{array}{l}f_{\mathrm{T}} \\
(\mathrm{GHz})\end{array}$ & $\begin{array}{l}f_{\max } \\
(\mathrm{GHz})\end{array}$ \\
\hline 53 & 440 & 530 & 30 & 44 \\
60 & 390 & 270 & 30 & 40 \\
65 & 510 & 500 & 40 & 52 \\
70 & 550 & 510 & 45 & 62 \\
75 & 370 & 310 & 36 & 47 \\
80 & 430 & 270 & 41 & 50 \\
85 & 400 & 420 & 33 & 51 \\
\hline
\end{tabular}

structures by standard photolithography and liftoff techniques. All the samples exhibited good pinchoff characteristics and output conductances in the range of 10 to $25 \mathrm{mS} / \mathrm{mm}$. The devices were biased near their peak $g_{\mathrm{m}}$ value for microwave measurements. From the measured $S$-parameter, the extrinsic values of $f_{\mathrm{T}}$ and $f_{\max }$ were extrapolated. Table 1 shows the summary of DC and microwave characteristics measured for each of the samples. The good DC and microwave results indicate the high quality of these layers.

The trend in the mobility data as a function of In content observed by us - an initial increase followed by a decrease at higher values of strain (In content) - has been observed by other authors [11]. Since the carrier mass is not expected to show a turnaround, to explain the mobility data it is important to examine other scattering mechanisms. The dominant mechanisms which could explain the decreased mobility at higher In composition are interface scattering and alloy scattering. For the GaAs based structures, the alloy scattering continuously increases as In composition $x$ is increased (since $\mu \propto 1 / x^{2}(1-x)^{2}$ ). However, for the InP based systems the alloy scattering should decrease since the alloy scattering peaks for a 50:50 alloy, assuming the mass does not vary. Thus unless some unusual clustering effects occur in strained epitaxy, one has to consider interface roughness as the source of the turnaround in mobility as the excess In composition increases beyond $-15 \%$. The interface roughness scattering can be described by the scattering rate [4]:

$\frac{1}{\tau}=\frac{\pi e^{4} m^{*}}{\hbar^{3} \epsilon_{\mathrm{s}}^{2}} N_{\mathrm{s}}^{2} \Delta^{2} \int_{0}^{\pi} J_{1}\left(k_{\mathrm{F}} L \sin \phi\right)^{2} \mathrm{~d} \phi$,

where $N_{\mathrm{s}}$ is the sheet charge density, $\Delta$ is the height of the $3 \mathrm{D}$ island describing the interface roughness, $L$ is the lateral extent of the island, and $k_{\mathrm{F}}$ is the Fermi vector. From the discussion section 2 we expect $\Delta$ to increase abruptly when the strain increases $\sim 2 \%$. We expect that if $\Delta$ increase to $\sim 4$ monolayers around this value of strain, the mobility decreases at high In content can be accounted for. The RHEED data of figs. 1c and $1 \mathrm{~d}$ suggest that this is quite likely the case. The existence of point defects is possible, but is difficult to identify. However, in-situ scanning tunneling microscopy (STM) data for $\mathrm{In}_{0.35} \mathrm{Ga}_{0.65}$ As on GaAs did clearly show that $3 \mathrm{D}$ growth occurs in the case of strained-layer epitaxy with large misfits. This supports our RHEED measurement results.

A MODFET grown by the MEE technique should suffer less from interface roughness scattering and, thus, have improved transport properties. To verify this argument, we have compared the properties of GaAs-based $\mathrm{Al}_{0_{3}} \mathrm{Ga}_{0.7} \mathrm{As} / \mathrm{In}_{0.2}$ $\mathrm{Ga}_{0.8}$ As MODFETs with $100 \AA \mathrm{In}_{0.2} \mathrm{Ga}_{0.8} \mathrm{As}$ channels. In one set the entire structure is grown by $\mathrm{MBE}$ at $560^{\circ} \mathrm{C}$ while in the other the last five monolayers of the $\operatorname{In}_{0.2} \mathrm{Ga}_{0.8}$ As channel is grown by MEE at $560^{\circ} \mathrm{C}$. The latter exhibits improved

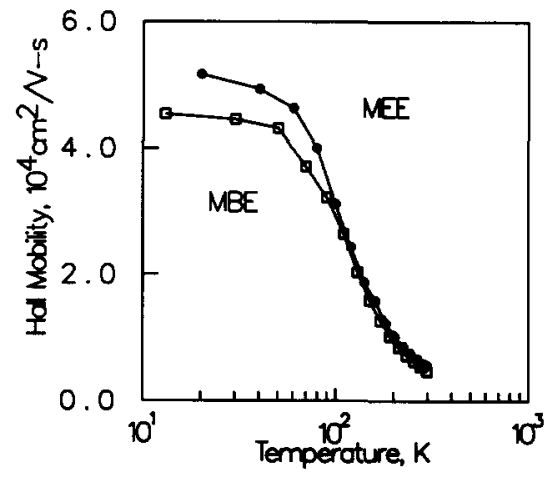

Fig. 5. Hall mobility as a function of temperature in $\mathrm{Al}_{0.3}$ $\mathrm{Ga}_{0.7} \mathrm{As} / \mathrm{In}_{0.2} \mathrm{Ga}_{0.8} \mathrm{As}$ MODFET structures grown by $\mathrm{MBE}$ and MEE. 
mobilities at temperatures ranging from 13 to 300 $K$ (fig. 5). The channel electron density in the two structures is $\sim 1.5 \times 10^{12} \mathrm{~cm}^{-2} .1 \mu \mathrm{m}$ gate MODFETs made of the MEE samples also show improved DC transconductance and microwave performance. For example, at room temperature $g_{\mathrm{m}}$ $=108$ and $59 \mathrm{mS} / \mathrm{mm}$ in the devices grown by MEE and MBE, respectively. Note that the low $g_{m}$ values are partly due to the low electron densities and part of the difference is due to process variation. In this MODFET structure, the modulationdoped layer has thickness of $150 \AA$ and nominal doping concentration of $2 \times 10^{18} \mathrm{~cm}^{-3}$. The spacer layer has thickness of $120 \AA$. This structure was intended for high electron mobility in the channel. For higher current capability and $g_{\mathrm{m}}$, we would increase the doping level and reduce the spacer thickness. We have also made $0.2 \mu \mathrm{m}$ gate $\mathrm{In}_{0.7} \mathrm{Ga}_{0.3} \mathrm{As} / \mathrm{In}_{0.52} \mathrm{Al}_{0.48}$ As MODFETs with the channel grown by MEE. The measured $g_{\mathrm{m}}$ and $f_{\max }$ are $570 \mathrm{mS} / \mathrm{mm}$ and $140 \mathrm{GHz}$, respectively, showing the suitability of the MEE technique for realizing high-performance devices.

\section{Conclusions}

In conclusion, we have addressed the issue of growth modes in MBE grown strained layers in the context of the performance characteristics and transport properties of pseudomorphic MODFETs. The advantages of MEE over MBE are also discussed. From RHEED oscillation measurements and energy minimization considerations it is apparent that once the misfit strain in the InGaAs channel increases above $-1.5-2.0 \%$, the growth occurs in a $3 \mathrm{D}$ island mode, leading to a rough interface. From RHEED oscillation studies we note that it is possible to achieve layer-by-layer growth even at high strain by MEE. MODFETS with the last several monolayers of the channel grown by MEE also exhibit better performance than those with entire structure grown by MBE.

\section{Acknowledgements}

The authors acknowledge help and comment provided by R. Lai and J. Pamulapati. This work was supported by the Office of Naval Research under Grant N00019-89-J1519.

\section{References}

[1] G.I. Ng, W.-P. Hong, D. Pavlidis, M. Tutt and P.K. Bhattacharya, IEEE Electron Device Letters EDL-9 (1988) 439.

[2] T.J. Drummond, T.E. Zipperian, I.J. Fritz, J.E. Schirber and T.A. Plut, Appl. Phys. Letters 49 (1986) 461.

[3] T. Ando, J. Phys. Soc. Japan 51 (1982) 3900.

[4] W.-P. Hong, J. Singh and P.K. Bhattacharya, IEEE Electron Device Letters EDL-7 (1986) 480.

[5] J. Singh and K.K. Bajaj, Superlattices and Microstructures 2 (1986) 185

[6] J.H. Neave, P.J. Dobson, B.A. Joyce and J. Zheng, Appl. Phys. Letters 47 (1985) 100

[7] J.M. Van Hove, P.R. Pukite, G.J. Whaley, A.M. Wouchak and P.I. Cohen, J. Vacuum Sci. Technol. B3 (1985) 1116.

[8] P.R. Berger, K. Chang, P.K. Bhattacharya, J. Singh and K.K. Bajaj, Appl. Phys. Letters 53 (1988) 684.

[9] Y. Horikoshi, M. Kawashima and H. Yamaguchi, Japan. J. Appl. Phys. 27 (1988) 169.

[10] F. Briones, L. Gonzalez and A. Ruiz, Appl. Phys. A49 (1989) 729.

[11] C.K. Peng, S. Sinha and H. Morkoç, J. Appl. Phys. 62 (1987) 2880. 